\title{
INVESTIGACIÓN PARTICIPATIVA CON AGRICULTORES: UNA OPCIÓN DE ORGANIZACIÓN SOCIAL CAMPESINA PARA LA CONSOLIDACIÓN DE PROCESOS AGROECOLÓGICOS
}

\author{
Gloria Inés Cárdenas Grajales ${ }^{1}$
}

\author{
Manizales, 2009-10-19 (Rev. 2009-11-06)
}

\begin{abstract}
RESUMEN
La participación es un método de investigación que permite a las comunidades apropiarse de herramientas y procedimientos para la toma de control y autonomía en procesos autogestionarios orientados a poner en práctica la agroecología; ello implica ir más allá del manejo técnico productivo de los agroecosistemas para situarse en perspectiva de construir formas de organización campesina. Sin embargo, en relación con las diversas maneras como los campesinos agricultores toman partido en una investigación, se dan diferentes niveles de participación, algunas de los cuales no representan opciones o alternativas metodológicas de construcción de poder local y de configuración de actores sociales y políticos, sino que se circunscriben a actividades operativas y funcionales que involucran parcialmente a los agricultores. Desde este punto de vista se hace necesario identificar cuáles son realmente los niveles de participación que configuran métodos de investigación, desde qué intereses y propósitos institucionales se plantean, qué alcances tienen, cuál es el papel de los campesinos investigadores y de los agentes externos que actúan en el proceso investigativo y hasta qué punto es posible lograr organización y autogestión comunitaria que poco responden a este propósito.
\end{abstract}

\section{PALABRAS CLAVE}

Investigación participativa, agroecología, organización social campesina, autogestión, poder local, autonomía, toma de decisiones.

\section{PARTICIPATIVE RESEARCH WITH FARMERS: A SOCIAL PEASANT ORGANIZATION OPTION FOR THE CONSOLIDATION OF AGROECOLOGICAL PROCESSES}

\begin{abstract}
Participation is a research method that lets communities adopt tools and procedures for control and autonomy in workers' self-management processes oriented towards agroecology. This implies going beyond the productive and technical management of agroecosystems, in order to build peasant organization forms. However, in relation to the diverse manners in which peasants participate in researches, different participation levels are reached, some of which do not represent methodological options or alternatives for the construction of local power and the configuration of social and political actors; instead their participation is circumscribed to manual and functional activities that partially involve the producers. From this point of view, it is necessary to identify the real participation levels that configure the research methods, starting from the institutional interests and purposes proposed, the role of peasant researchers and external agents active in the research process, and up to what point is it possible to achieve community organization and workers' self-management that don't necessarily respond to this purpose.
\end{abstract}

KEY WORDS: Participative research, agroecology, social peasant organization, workers' selfmanagement, local power, autonomy, decision making.

\section{INTRODUCCIÓN}

Las organizaciones campesinas que han buscado opciones alternativas para manejo de sus agroecosistemas han emprendido en los últimos veinte años procesos de desarrollo rural enmarcados en el enfoque agroecológico. Muchas de estas organizaciones han aplicado metodologías participativas y han operado desde su propia dinámica organizativa procesos de cambio y transformación no sólo de sus agroecosistemas sino también de su formación como actores políticos y económicos. De ello dan cuenta experiencias exitosas de agroecología que existen en el país cuya evolución no ha sido 
suficientemente visibilizada y documentada, tras estos procesos se pueden evidenciar investigaciones participativas que tienen un doble propósito: por un lado la organización comunitaria para la toma de decisiones y por el otro, la construcción de sujetos políticos con capacidad de organización y movilización social, el fortalecimiento de esquemas internos de organización comunitaria y de toma de decisiones en aspectos relacionados con el manejo de sus fincas, la generación de condiciones de seguridad y soberanía alimentaria, la conservación de sus bienes naturales (agua, suelos, biodiversidad), el acceso a salud, educación y vivienda digna para sus familias y la consolidación de propuestas autónomas de comercialización local e internacional.

La investigación participativa no solamente es una herramienta para que los agricultores apoyen investigaciones en ensayos experimentales, sino que también se constituye en un escenario posible para lo que Freire llama el proceso cíclico de concientización, acción, educación y que constituye, junto con la agroecología, una alternativa de configuración de nuevos escenarios para las sociedades rurales.

Este artículo aborda algunas consideraciones sobre la importancia de la investigación participativa llevada a cabo por organizaciones campesinas en la consolidación de proyectos con enfoque agroecológico, y en qué se diferencia su aplicación desde los fines de la investigación agroecológica y otras formas de investigación en las ciencias agrícolas.

\section{LA INVESTIGACIÓN PARTICIPATIVA EN AGROECOLOGÍA}

Desde una comprensión holística de la agroecología se hace necesario el abordaje de dinámicas participativas a partir de los propios agricultores para generar investigaciones, transformaciones y cambios que respondan directamente a sus intereses, donde la actividad investigativa deje de estar en manos exclusivas del investigador para dar paso a la realización de actividades conjuntas investigadoragricultor y al diseño participativo de acciones productivas y de cambio social que mejoren su nivel de vida (Sevilla Guzmán,

2004).

La investigación participativa implica que los agricultores adquieran el papel de investigadores, tomando responsabilidad e iniciativa de las acciones como conductores del curso dinámico de sus prácticas donde el problema a investigar es definido, analizado y resuelto por los propios grupos sociales, desde sus lógicas y cosmovisiones.

La participación, como metodología para avanzar hacia la construcción de organización campesina, es considerada junto con la agroecología, una manera de resignificación de la realidad social campesina como punto de partida de todo proceso de reconversión de la agricultura, la organización social, la educación popular y el desarrollo endógeno.

En la conexión entre la investigación participativa y la agroecología se hacen pertinentes los criterios esbozados por Orlando Fals Borda, quien en el año 1998 vio la participación como un nuevo paradigma en investigación que se mueve alrededor de algunos criterios claves que él propuso y que son importantes de esbozar para los fines de este documento: - "en la metodología de la IAP es clave la recuperación histórica" de los procesos comunitarios, -es fundamental aplicar el criterio de "una ciencia modesta... las técnicas de investigación no deben ser sofisticadas ni complicadas, para que las personas que no hayan ido a la universidad sean capaces de dominarlas y aplicarlas", - en cuanto al lenguaje, la investigación participativa debe aplicar el principio de "devolución sistemática del conocimiento a las bases", por último esboza que "la investigación participativa así como la educación popular tienen políticos inevitables" (Fals,

La investigación participativa surge en el marco de nuevas concepciones del desarrollo rural en América Latina, en éstas se amplían las consideraciones acerca de la mitigación de la pobreza y se fomenta una orientación hacia una visión de lo regional, con un manejo del concepto de sostenibilidad, no sólo de los recursos naturales, sino también económica, política, social y cultural donde se incorpora el concepto de empoderamiento de las comunidades campesinas en la búsqueda de que los pobladores rurales y las distintas organizaciones se doten de poder para que puedan ejercitar sus derechos frente al estado, estas perspectivas incorporan el concepto de participación de los distintos actores sociales en los diferentes procesos y proyectos (Pérez y Farah, 2004). 
En los países latinoamericanos esto ha ido de la mano con los procesos de descentralización político administrativa y con la creación de instancias de participación a nivel local, regional y nacional; esta orientación ha conducido a una metodología de intervención de los actores de abajo hacia arriba hacia la confluencia de intereses para el logro del desarrollo local.

La investigación participativa surge como una alternativa a los métodos de investigación que tradicionalmente se han aplicado en las ciencias agropecuarias, se sustenta como opción válida para transformar la realidad y obtener nuevos conocimientos pertinentes y contextualizados a las necesidades e intereses de los agricultores. Méndez y Gliessman 2002 plantean la integración de la agroecología con enfoques de las ciencias sociales, como base para la investigación interdisciplinaria en relación con el manejo de los recursos naturales y el desarrollo rural en el trópico latinoamericano o neotrópico. La agroecología integra conceptos ecológicos al manejo de los ecosistemas, siendo un buen punto de partida para promover procesos innovadores en los paisajes rurales. La agroecología debe acompañarse de varios enfoques promisorios desde las ciencias sociales para enfrentar la compleja dinámica social y ambiental que se presenta en las áreas rurales de los países en desarrollo.

Los enfoques participativos con pequeños agricultores tienen sus orígenes en la sociología, la antropología, el desarrollo rural y la investigación participativa, estos enfoques junto con la investigación y desarrollo participativo dirigidos al manejo de los propios de los modelos tradicionales de investigación y desarrollo rural (Méndez y Gliessman, 2002).

Estos autores muestran el avance que han tenido la investigación y el desarrollo participativos al incluir aspectos de monitoreo y evaluación desde la perspectiva del agricultor experimentador; a pesar de esto, muchas de las instituciones, ONGs y agencias de financiación han puesto en controversia el nivel de participación que realmente obtienen las comunidades y su efecto a mayores escalas políticas, aquellas donde se definen decisiones de importancia para las mismas comunidades.

La investigación participativa con agricultores en el marco del conocimiento agroecológico representa una alternativa de integración y acercamiento entre el conocimiento tradicional y el conocimiento científico convencional; desde este punto de vista se plantean modos de relación entre agricultores y profesionales del campo a partir del diálogo de saberes y del reconocimiento y respeto por el saber tradicional de las comunidades rurales y la forma de relación con la naturaleza; desde este enfoque se da particular importancia a la horizontalidad en la relación agricultor - profesional. "Esto implica un diálogo mediante la comunicación intercultural revalorizadora, para dinamizar el saber de la comunidad rural tal como es, así se aproxima al punto de vista en que se desenvuelve el actor orientado como agente del sistema social" (San Martín, 2002).

Esta forma de investigación toma elementos del enfoque histórico cultural y los métodos de investigación cualitativa; pero lo que define sus alcances son los niveles de participación que los agricultores alcanzan a través de proyectos de desarrollo en los que las comunidades se organizan, diagnostican, planifican, ejecutan y evalúan sus situaciones particulares con miras a un desarrollo rural endógeno. Un aspecto importante de la investigación participativa lo constituyen los procesos de organización comunitaria que son requisito importante a la hora de llevar a cabo este tipo de investigaciones. La condición cíclica de reflexión y acción como estrategia de solución a diversas problemáticas de las comunidades rurales, representa una alternativa importante para lograr participación y organización local de las mismas como un método que permite la concienciación y acción sobre sus problemáticas.

A pesar de las críticas a estos enfoques desde la forma convencional de abordaje de la investigación en los sistemas de producción, este documento parte de la base del gran potencial que tienen los enfoques crítico-sociales como herramienta para la investigación y el desarrollo en el manejo de los sistemas productivos campesinos. La investigación por sí sola no garantiza la solución a los problemas de los agricultores, se requiere de la complementación con otras herramientas y procesos que permitan la continuidad y la permanencia de las acciones, como el empoderamiento, la toma de decisiones desde lo local y la construcción de sujetos políticos. Para ello se hace necesario llegar a entender las realidades de las comunidades y de los agricultores a nivel local: esta comprensión requiere de tiempo y de la generación de niveles de confianza con los actores rurales locales.

En la construcción de organización social campesina desde el enfoque agroecológico, deben tenerse en cuenta aspectos tales como: - la realidad campesina como punto de partida de todo proceso de desarrollo, participación y educación, - el conocimiento entendido como la toma de conciencia y 
condicionado a la práctica social, - la participación de los sujetos sociales en la transformación de su realidad social como una garantía de toma de conciencia, - el desarrollo de capacidades en la toma de decisiones y la organización, - el desarrollo de habilidades de discernimiento y debate, - la generación de procesos de identidad cultural, - la articulación de prácticas sociales entre la educación y lo económico productivo, - la incorporación de procesos de reflexión acción en relación con las problemáticas comunitarias y la realización de acciones colectivas alrededor de las mismas (Secretaría de Desarrollo de la Comunidad, Gobernación de Antioquia, 1994).

Son muchos los autores que desde diversas disciplinas sustentan los aportes que puede hacer la investigación participativa al reconocimiento y valoración de las experiencias de los productores locales y la recuperación de los conocimientos tradicionales asociados a ella; la agroecología reconoce en la investigación participativa un principio fundamental que sustenta el "diálogo de saberes", volviéndose un objetivo fundamental de la investigación agroecológica; bajo este concepto, la investigación participativa aporta a la construcción de los fundamentos y métodos científicos de una agricultura alternativa (Altieri, 1995) en donde se valoren y rescaten los saberes locales para mantener y acrecentar la variedad genética, los policultivos (agrícolas, forestales, agroforestales), la diversidad de prácticas productivas, y por último, la heterogeneidad paisajística, lo que contribuye a mantener una cierta sustentabilidad, basada en la resiliencia. (Toledo, 2005)

La investigación participativa parte del entendimiento y valoración del saber tradicional y el papel que en la conservación de los recursos naturales han jugado las comunidades a través del tiempo; esta premisa pone a los profesionales del sector agropecuario en la posición de replantear su conocimiento como único y valedero, y por ende la forma de comunicación que se tiene con el agricultor. A partir de este entendimiento se redimensiona el papel de comunicador único para dar paso a un proceso más consensuado que privilegia el trabajo en equipo y el aporte que hacen los mismos agricultores a la gestión de sus agroecosistemas. "...las metodologías participativas han mostrado su viabilidad en descubrir y entender el conocimiento y el saber local (ya sea en torno a lo productivo como a lo cultural) como claves para la sustentabilidad... así como para fortalecer el empoderamiento de los actores marginados... asegurando así su validación como sujetos de derechos y actores de su desarrollo" (Contreras, 2002).

Geilfus (2001) afirma que la participación no debe limitarse a eventos de consulta y planificación, los procesos deben trascender esta tendencia para crear una dinámica en la cual cambien los papeles respectivos del profesional: técnico o promotor facilitador, y de los miembros de la comunidad. De acuerdo con este autor "en la actualidad el esquema verticalista de desarrollo es caracterizado por el uso de métodos de investigación que extraen la información de la gente sin su participación consciente, sobre la base de estas informaciones se toman decisiones en las cuales la gente casi nunca toma parte".

En la formulación de propuestas de investigación participativa deben tenerse en cuenta problemas como la falta de un lenguaje común entre técnicos y comunidades y el cambio de papel del extensionista tradicional a facilitador del desarrollo. El rol del profesional inmerso en la dinámica de las sociedades rurales apostándole a una propuesta agroecológica, debe ser el de facilitador de procesos, en ese cambio de rol se debe tener en cuenta un cambio de actitudes y un intercambio de informaciones entre todos los actores, donde se privilegien metodologías de investigación activas y enfoques más horizontales de revalorización del saber. Un facilitador es un profesional que comparte experiencias con los agricultores, apoya a la comunidad en el diagnóstico de sus problemas y en la planificación de sus predios, parte de las necesidades de la propia gente y busca las concertaciones y negociaciones más adecuadas; pero sobre todo, mantiene una comprensión y respeto por las lógicas y cosmovisiones campesinas, además de vigilar permanentemente su intervención como actor externo para no caer en las mismas prácticas de la extensión agrícola en su forma de hacer transferencia de tecnologías de los investigadores a los agricultores.

El reencuentro con la agroecología por parte de las comunidades campesinas implica ciertos niveles de acompañamiento y aporte de las instituciones y profesionales del sector agropecuario pero demanda también liderazgo y compromiso de las comunidades frente al papel que deben desempeñar en la toma de decisiones y en la organización local; en este esquema el rol del profesional cambia del extensionista rural tradicional al de facilitador y acompañante de procesos, lo que implica el desarrollo de competencias para la organización, el trabajo en equipo y la sistematización. 
Sevilla Guzmán (2004) ubica la investigación agroecológica en tres niveles o perspectivas que corresponden a un pluralismo transdisciplinar y epistemológico: la ecológico-productiva, la socioeconómica de acción local y la sociopolítica de transformación social. Se resalta la importancia de la perspectiva socioeconómica, "a la cual le corresponde promover dinámicas participativas desde los propios productores, incorporando metodologías participativas y dotando de un sentido sociocultural los procesos generados por estos, sean naturales o sociales". El autor plantea igualmente como "juega papel importante en este enfoque la explicación de los fenómenos desde la percepción de los sujetos intervinientes en los mismos y los discursos elaborados por estos", los cuales en agroecología se dan inmersos en el proceso productivo y vinculados al manejo de los recursos naturales, "mostrando así la necesidad de incorporar técnicas participativas para obtener un manejo ecológico de los recursos naturales".

Desde la perspectiva de transformación social, Sevilla Guzmán (2004) plantea la necesidad de dotar las actividades productivas, socioeconómicas y culturales de los campesinos agricultores, de un contenido sociopolítico que implica actividades conjuntas investigador- agricultores para el diseño participativo de acciones productivas y de cambio social que mejoren niveles de vida, para ello, "con los procesos de investigación participativa no sólo se trata de dar voz a lo investigado (al reconocer la lógica de sus prácticas productivas), sino de aceptar que éste adquiera el papel de investigador (tomando responsabilidad e iniciativa de las acciones) en la generación de redes de intercambio y estrategias de acción productivas vinculadas a los movimientos sociales rurales".

\section{ENFOQUES}

Actualmente ocurre que la investigación participativa se ha convertido en una herramienta utilizada por los centros de investigación agropecuaria, varios de ellos afiliados a la red CGIAR -Grupo Consultivo para la Investigación Agrícola Internacional- , para fomentar la participación de los agricultores en procesos de investigación, lo que implica que sean capacitados "como agentes de extensión y/o facilitadores del desarrollo (dependiendo de las intenciones y propósitos de la investigación) tanto para proporcionar asistencia técnica como para promover la innovación y la experimentación entre los agricultores" (Hellin, et al. 2006); esto implica una participación parcial de los agricultores en procesos de investigación de corte experimental donde sus funciones están relacionadas con la recepción de información acerca de los proyectos, la capacitación para hacer seguimiento y monitoreo y la participación en la transferencia y adopción de los resultados de los ensayos experimentales en sus propias parcelas.

Sin embargo, la investigación participativa va más allá de la participación funcional o por estímulos. Lo que se busca es el logro de la organización, la autogestión y la autonomía comunitaria, y de acuerdo con (Hellin, et al., 2006) "el fortalecimiento de las capacidades locales y en el caso de los individuos que participan en el proceso, la generación de mayor confianza en sí mismos y en sus conocimientos", lo cual implica procesos de mayor alcance en el tiempo y en los resultados, es decir, una participación más funcional e interactiva hasta llegar al auto-desarrollo (Geilfus, 2001).

El carácter de la participación y el grado de intervención de la comunidad en el proceso de investigación participativa, marca diferencias y gradualidades en lo que se ha denominado Investigación Acción Participación. Cuando el proceso tiene como propósito la aplicación de una serie de pasos planificados y diseñados por un equipo científico técnico, "que, a partir de un diagnóstico de la realidad comunitaria diseña la investigación, sus objetivos y el método de la misma, incluyendo la participación parcial de la comunidad, ya sea para la recolección y/o contrastación de los datos de investigación, o para la implementación de estrategias a seguir, donde los resultados del proceso investigativo son ordenados, sistematizados e interpretados por el equipo de investigación, pudiendo ser devueltos a la población estudiada" (Moreno y Espadas, 1998) estamos hablando de una forma de investigación participativa donde el interés de los investigadores es que los agricultores campesinos suministren y recaben información, son consultados y reciben incentivos por su actividad en el proyecto, además se convierten en "agentes de extensión" para transferir las tecnologías derivadas del proyecto a otros agricultores mediante equipos de investigación campesina, comités agrícolas locales, escuelas de campo, investigadores locales y otras figuras ideadas para este fin desde la investigación agrícola convencional.

Por otro lado, la investigación participativa desde la visión de un proceso de Investigación Acción Participación, "concede un carácter protagónico a la comunidad en la transformación social que 
necesita, el problema a investigar es delimitado, atendido, analizado y confrontado por los propios afectados. El rol de investigador vendría a ser el de dinamizador y orientador del proceso, con lo que se tendería a revertir la dicotomía sujeto-objeto, produciéndose una relación de cohecho entre el grupo o comunidad y el equipo de investigación" (Moreno y Espadas, 1998).

La situación anterior ha derivado en visiones distintas de lo que debe ser la investigación participativa la cual se asume con diversas intencionalidades de acuerdo con las instituciones que la aplican y los intereses en su utilización. El nivel y el objetivo de la participación de las comunidades en el proceso de investigación es para Moreno y Espadas, 1998, el "eje diferenciador de ambas estrategias investigativas, ya que la IAP parte del supuesto de que la participación no puede confundirse con una simple preocupación por encontrar apoyo reflexivo de los implicados, para los objetivos del conocimiento e intervención, delineados por un equipo exterior al grupo o comunidad". La IAP no debe confundirse con procesos de intervención y desarrollo exógenos donde se utilizan metodologías participativas que traen consigo bajos niveles de construcción de organización social campesina, la IAP tiene implícita en sus dimensiones de investigación, acción, participación la condición de transformación y abordaje de la realidad social a partir de la producción de conocimiento por parte los propios sujetos, en este caso los campesinos agricultores, quienes además de los facilitadores, se ponen en presencia de un "nuevo paradigma científico bajo la condensación de un conjunto particular de condiciones históricas, como afirma Kunt, estamos en presencia de un desarrollo análogo: el de una particular metodología (o tecnología blanda) que revolucionó nuestra forma de hacer y concebir la ciencia" (Falabella, 2002).

Los centros de investigación han hecho una sistematización importante de lo que ha sido y debe ser la investigación participativa con agricultores desde los intereses funcionales que cumplen los agricultores en investigaciones de corte experimental. De estas experiencias existen publicaciones importantes, sin embargo son poco conocidos los procesos de investigación participativa con enfoque de construcción de organización social campesina que han realizado las organizaciones campesinas a partir de la consolidación de sus proyectos de organización comunitaria con enfoque agroecológico y del aporte que a estos procesos han hecho las herramientas participativas.

La investigación participativa es una forma de hacer investigación que está siendo ampliamente utilizada para lograr que los agricultores no solamente participen de manera tangencial en los proyectos de desarrollo sino que también avancen hacia dinámicas de organización y autogestión para la solución de las múltiples problemáticas que se presentan a los agricultores de economía campesina, sin embargo estos dos aspectos deben estar muy claros para las comunidades a la hora de convocar su participación en proyectos de esta índole.

La claridad sobre los tipos de objetivos y participación de una investigación con campesinos agricultores debe darse, sin lugar a dudas, porque en cada uno de los tipos de investigación participativa descritos, el nivel de compromiso y acción de las comunidades es diferente: mientras en una es muy posible que la participación de los agricultores sea parcial y por estímulos, y con ello se sigan replicando los esquemas de asistencialismo y paternalismo con los que se ha intervenido a las comunidades rurales, en la otra se requieren altos niveles de compromiso, organización social local y capacidad para la toma de decisiones e inserción en espacios y políticas públicas.

Las intenciones anteriormente explicitadas surgen en el marco del reconocimiento de la autonomía que deben tener las organizaciones campesinas de base, en relación con las posibilidades que la organización y la participación a nivel local ofrece.

En este sentido De Grammont (2006) plantea que "la autonomía de acción y el arraigo local para solucionar demandas concretas son la base de la participación, pero la unidad de acción no pasa por la creación de organismos centralizadores sino por la elaboración de un proyecto alternativo de sociedad. El reto para las organizaciones de productores, no es sólo cómo reconstruir sus proyectos desde abajo, sino cómo vincular sus proyectos locales, por esencia parciales, en un modelo nacional de desarrollo con equidad y justicia social, y sustentable. Esto supone pensar la reconstrucción desde abajo, sin duda, pero siempre buscando la articulación de lo local con lo nacional". 


\section{CONCLUSIÓN}

El impacto de la investigación y la participación en las sociedades rurales resulta de las posibilidades que estas herramientas ofrecen a los procesos de organización social, en cuanto permiten visibilizar acciones colectivas de investigación campesina diferentes de la investigación agrícola convencional, y comprender además hasta qué punto es posible que la participación pueda ser realmente un proceso autónomo de las comunidades y a partir de ella se puedan generar las condiciones para la conservación de esta autonomía, en este sentido es importante la puesta en crisis de los siguientes aspectos:

- El doble sentido de la participación popular: en tanto, según de Grammont, "es positiva si posibilita una mayor participación, pero es negativa en la medida en que a menudo fortalece el poder de las burguesías

- La posibilidad de la participación de configurar realmente actores sociales. - La participación como opción del campesinado para construir escenarios que permitan a partir del arraigo y la autonomía seleccionar y apropiarse de sus problemáticas, no desde la creación de organismos centralizadores, sino desde sus propios proyectos alternativos de sociedad. - El reto de una participación que sea capaz de incluir sus proyectos locales en un modelo de desarrollo agrícola equitativo e incluyente. - La autonomía de la participación frente a esquemas "parlamentarios" con lo cual existe una constante tensión en tanto que se requiere fortalecer la participación popular pero a su vez se busca apropiación por parte de esquemas convencionales "parlamentaristas" con el ideal de la "representación" de unas cúpulas que terminan por vulgarizar y deformar el concepto mismo con sus prácticas clientelistas, burocráticas y engañosas. - La utilización de esquemas de investigación convencional que utilizan el discurso de la participación para que los agricultores realicen funciones de operarios y auxiliares de investigaciones bajo la figura deformada de la participación.

\section{BIBLIOGRAFÍA}

- AltieRI, M.A. Agroecología: principios y estrategias para diseñar sistemas agrarios sustentables. Ediciones Científica Americanas. Pág. 27-34

- DE GRAMMONT, HUBERT C. (Compilador). La Construcción de la Democracia en el Campo Latinoamericano. CLASCO. Consejo Latinoamericano de Ciencias Sociales. Bueno Aires Argentina, 2006. Pág. 9-21, 132-155

- CONTRERAS O. R. La Investigación Acción Participativa (IAP): revisando sus metodologías y potencialidades. En: Experiencias y Metodologías de la Investigación Participativa, John Durston y Francisca Miranda, CEPAL Serie Políticas Sociales No.58, Santiago de Chile, 2002, Pág. 11-12

- FALS B.,O. Investigación Acción Participativa Aportes y Desafíos, 7 Ed., Santafé de Bogotá, Dimensión Educativa, 1998. ISSN: 0122-8773, 72

- Falabella, G. Investigación Participativa: nacimiento y relevancia de un nuevo encuentro ciencia sociedad. En: Experiencias y Metodologías de la Investigación Participativa, John Durston y Francisca Miranda, CEPAL Serie Políticas Sociales No.58, Santiago de Chile, 2002, Pág. 22-23

- GEILFUIS, F. Ochenta Herramientas para el Desarrollo Participativo, 3 Ed., Bogotá: Editorial Kimpres Ltda., 2001, ISBN: Vol: 958-96964-2-2 OBRA: 958-331663-6. 120 p.

- GliESSMAN, S. R. Agroecología: Procesos Ecológicos en Agricultura Sostenible. Litocat: Turrialba, Costa Rica, 2002 ISBN 9977-57-385-9, Pág. 17-27

- hellin, J., BELlon, M., BADSURE, L. Reduciendo la brecha entre la realidad de los investigadores y la de los agricultores. En: Leisa: Revista de Agroecología: Investigación Participativa y Desarrollo. Vol. 22 No.3, 2006, pag.5-8

- Hellin, J., DE LA TORRE, C., COELLO, J. RODRígueZ, D. Los Kamayoq en el Perú: expertos campesinos para la extensión y la experimentación. En: Leisa: Revista de Agroecología: Investigación Participativa y Desarrollo. Vol. 22 No.3, 2006, pag.5-8

- MÉNDEZ V.E., Y S.R. GLIESSMAN. 2002. Un enfoque interdisciplinario para la investigación en agroecología y desarrollo rural en el trópico Latinoamericano. En: Manejo Integrado de Plagas y Agroecología. No.64: 5-16. 
- MORENO JL. y ESPADAS M. Investigación Acción Participativa.1998 En: Experiencias y Metodologías de la Investigación Participativa, John Durston y Francisca Miranda, CEPAL Serie Políticas Sociales No.58, Santiago de Chile, 2002, Pág. 22-23

- PÉREZ, C. E. MAYA, V.D. Y FARAH Q. M. A. Metodologías Participativas en la formulación y planificación de proyectos de desarrollo rural. Fase de diagnósticos en siete municipios del sur del Huila. En: Cuadernos de Desarrollo Rural. No. 47, 2001. Pág. 99 - 113

- SAN MARTíN, M.J. Participación de los actores en la implementación de Proyectos de Desarrollo". En: Metodologías Participativas hacia el diálogo de saberes. Maela: Movimiento Agroecológico Latinoamericano.2002. Pág. 13-20

- S.A. Investigación Acción Participativa Aportes y Desafíos, 7 Ed., Santafé de Bogotá, Dimensión Educativa, 1998. ISSN: 0122-8773, 72 p.

- SECRETARÍA DE DESARRollo DE LA COMUnidAD, Elementos Conceptuales para el Trabajo Comunitario. Gobernación de Antioquia, 1994.

- SEVILLA G.E. La Agroecología como Estrategia Metodológica de Transformación Social. Documento Electrónico. Instituto de Sociología y Estudios Campesinos de la Universidad de Córdoba. España, 2004.

- $\quad$ TOLEDO V.M. La Memoria Tradicional: La importancia agroecológica de los saberes locales. En: LEISA Revista de Agroecología: Ecoagricultura cultivando con la Naturaleza. Abril 2005, Vol.20 N.4: 16-19.

1. Administradora de Empresas Cooperativas, Especialista en Agroecología Tropical Andina, estudiante de la Maestría en Sociedades Rurales del Departamento de Desarrollo Rural de la Facultad de Ciencias Agropecuarias Universidad de Caldas. gloincasa@yahoo.es 